\title{
Die Besonderheiten der Ernährung im Leistungssport - von Freizeit- bis zu Hochleistungsaktivitäten
}

\author{
Characteristics of Nutrition in Competitive Sports, Ranging from Leisure \\ Activities to High-Performance Athletics
}

Autor

Institut

\section{H. Braun}

Institut für Biochemie, Momentum - Deutsches Forschungszentrum für Leistungssport, Deutsche Sporthochschule Köln

\author{
Schlüsselwörter \\ - Energieverfügbarkeit \\ - Energieumsatz \\ - Proteinbedarf \\ - Kohlenhydratzufuhr \\ - Trainingsbelastung \\ - Low-Glykogen-Training \\ - Low Carb

\section{Keywords} \\ - energy availability \\ - energy expenditure \\ - protein requirement \\ - carbohydrate intake \\ - exercise load \\ - low-glycogen training \\ - low carb
}

\section{Bibliografie}

DOI http://dx.doi.org/

10.1055/s-0042-102718

Aktuel Ernahrungsmed 2016;

41, Supplement 1: S10-S14

(c) Georg Thieme Verlag KG

Stuttgart · New York

ISSN 1862-0736

\section{Korrespondenzadresse}

Hans Braun

Institut für Biochemie, Deutsche

Sporthochschule Köln

Am Sportpark Müngersdorf 6

50933 Köln

Tel.: 0221/4982-4932

h.braun@dshs-koeln.de

\section{Zusammenfassung}

$\nabla$

Die Ernährung hat erheblichen Einfluss auf die körperliche und mentale Leistungsfähigkeit und ist für Leistungssportler eine wichtige trainingsbegleitende Maßnahme. Es handelt sich dabei jedoch nicht um eine Ernährungsform, die für alle Sportler in jeder Situation gültig ist. Die Frage nach der optimalen Ernährung benötigt eine Auseinandersetzung mit der Sportart, der aktuellen Trainingsphase, den individuellen Bedürfnissen und Zielen der Athleten. Die Umsetzung braucht Zeit, die Eigenmotivation der Sportler und des Fachpersonals, das sich intensiv mit den Ernährungsbedürfnissen von Sportlern auseinandersetzt. Neben einer bedarfsgerechten Energiezufuhr kommt es darauf an, die Energie sinnvoll auf die Energieträger Kohlenhydrate, Fette und Protein zu verteilen. Der erhöhte Proteinbedarf bei Leistungssportlern kann in der Regel mit der normalen Ernährung gedeckt werden; Supplemente sind nur in Ausnahmefällen erforderlich. Studien zufolge sind kleine Mengen von 15-25g Protein nach dem Krafttraining sinnvoll, um die Muskelproteinsynthese anzuregen. Der Bedarf an Kohlenhydraten steigt dynamisch mit der Intensität und Dauer der körperlichen Belastung. Eine ausreichende Versorgung ist entscheidend, um maximale Leistung abrufen zu können. Low Carb-Diäten sind für Leistungssportler eher ungeeignet. Dagegen kann ein sog. Low-Glykogen-Training in ausgewählten Trainingsphasen zu verbesserten Anpassungsprozessen führen und die Leistungsfähigkeit steigern.

Sportmediziner unterscheiden 4 Kategorien von Sportlern [1]: Breitensportler betreiben vor allem Sport aus Freude an der Bewegung, Gesundheitssportler möchten die präventiven und rehabilitativen Aspekte des Sports nutzen. Für diese beiden Gruppen gelten hinsichtlich Anpassung und Trai-

\section{Abstract \\ $\nabla$}

Nutrition has a crucial influence on physical and mental performance ability and is an important measure along sidetraining in high-performance athletes. However, this form of nutritionis not applicable for every athlete and in every situation. The question of optimal nutrition requires involvement with the particular type of sports, an athlete's current training stage, and athletes' individual requirements and objectives. Implementation takes time and individual motivation on the part of athletes and the specialist staff who engage intensively with the nutritional needs of athletes. In addition to adequate energy provision, it is important to divide the energy sensibly among the energy sources carbohydrates, fats, and protein. Performance athletes' higher need for protein can usually be covered in their regular diet; supplements are needed only in exceptional cases. Studies have shown that small amounts of $15-25 \mathrm{~g}$ protein are sensible after weight training, in order to stimulate muscle protein synthesis. The need for carbohydrates increases dynamically with the intensity and duration of physical exertion. A sufficient supply is crucial for achieving maximum performance. Low carb diets are unsuitable for performance athletes. So called low-glycogen training, however, can lead to better adjustment/adaptation processes in selected training stages and can increase performance ability.

ningsleistung die allgemeinen Empfehlungen der Deutschen Gesellschaft für Ernährung (DGE) für einen abwechslungsreichen, vollwertigen Speiseplan.

Leistungssportler trainieren dagegen systematisch mit dem Ziel, überdurchschnittliche Leistun- 

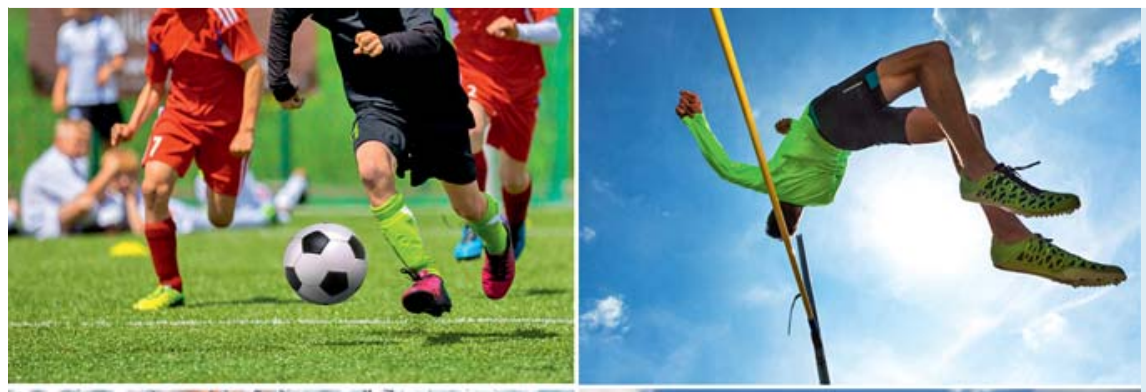

Abb. 1 Sporternährung trägt dazu bei, die Leistung des Athleten zu optimieren. Um die individuellen Besonderheiten zu erarbeiten, ist es notwendig, sich mit der jeweiligen Sportart und deren Trainingsbelastungen intensiv auseinanderzusetzen (Quelle: fotolia, ( ) matimix, () Westend61, ( ) Stefan Schurr, @ Valeriy Velikov).
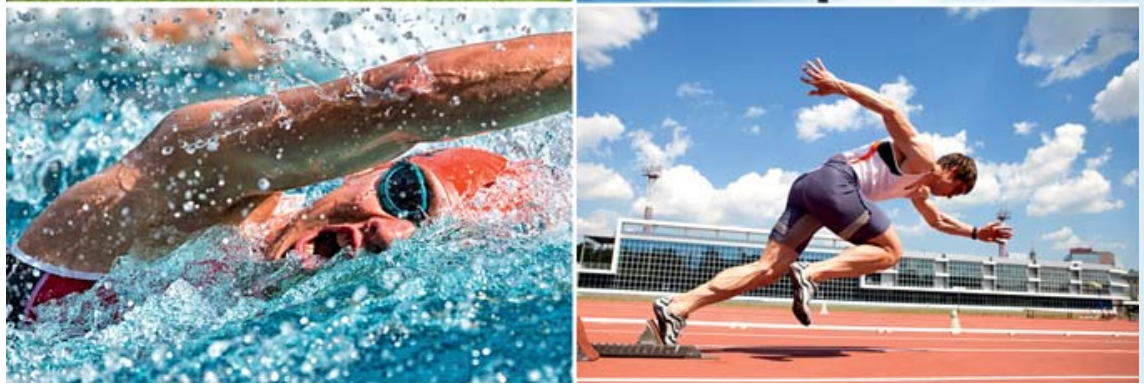

gen zu erbringen. Hochleistungssportler investieren sehr viel Zeit in den Sport mit dem Ziel, an nationalen und internationalen Wettkämpfen teilzunehmen [1]. Für diese Gruppen sind individuell ausgearbeitete Ernährungsstrategien wichtig, um die Leistungsfähigkeit zu erhalten und zu verbessern [2].

\section{Sporternährung funktioniert nicht nach Schema F $\nabla$}

Sportler sind keine genormte Zielgruppe, sondern unterscheiden sich in ihren Voraussetzungen, sowohl genetisch als auch in ihrem Entwicklungspotenzial. Entsprechend differenziert sind die Anforderungen an die Ernährung: Die Strategie richtet sich nach der Sportart, der aktuellen Trainingsphase, den Vorgaben und Zielen der Athleten [2] ( $\bullet$ Abb. 1).

Bei Hochleistungssportlern geht es einerseits um die sportlichen Ehren, aber auch um ökonomische Fragen: Manchmal entscheiden Bruchteile von Sekunden über den ersten und zweiten Platz und damit über große Unterschiede im Preisgeld. Auch aus diesem Grund sollten Athleten an der Frage interessiert sein, wie Ernährung dazu beitragen kann, die Leistungsfähigkeit zu maximieren.

\section{Professionelle Beratung}

Das 2011 veröffentlichte Konsensus-Statement „Sporternährung“ des Internationalen Olympischen Komitees erklärt, dass Ernährung die Leistungsfähigkeit beeinflusst und empfiehlt Athleten vor, während sowie nach dem Training und Wettkampf angepasste Ernährungsstrategien [2]. Dabei geht es nicht nur um die körperliche, sondern auch um die mentale Leistungsfähigkeit. Das Konsensus-Statement betont, dass Athleten mit professionellen Fachkräften zusammenarbeiten sollten, um sich in punkto Energie-, Nährstoff- und Flüssigkeitsbedarf beraten zu lassen. Damit sind Fachkräfte mit einer fundierten Ausbildung gemeint, wie Ernährungswissenschaftler und Oecotrophologen. Dabei ist ein sportwissenschaftlicher Hintergrund sehr hilfreich, denn die Planung einer optimalen Strategie für Training, Wettkampf und Erholungsphase erfordert profundes Grundlagenwissen und die intensive Auseinandersetzung mit einer Sportart und der individuellen Situation eines Athleten.

Wichtige Voraussetzungen für sportliche Erfolge sind in erster Linie Talent, Training, Motivation und Taktik. Eine optimale Ernährung kann diese Faktoren nicht ersetzen; sie wird aus einem untrainierten Sportler keinen erfolgreichen Sportler machen. Umgekehrt gilt aber: Der trainierte Sportler wird aufgrund einer nicht angepassten Ernährung nicht in der Lage sein, sein Leistungspotenzial im Training und Wettkampf abzurufen.

\section{Große Unterschiede im Energiebedarf \\ $\nabla$}

Wie differenziert die Ernährung von Spitzensportlern geplant werden muss, zeigen schon die großen Unterschiede im Energiebedarf, die je nach Training und Wettkampfkalender von Tag zu Tag und innerhalb der Saison variieren können. Kunstturnerinnen oder Marathonläufer beispielsweise wiegen nur ca. $50 \mathrm{~kg}$, Diskuswerfer, Kugelstoßer oder Gewichtheber können dagegen über $120 \mathrm{~kg}$ auf die Waage bringen und müssen dabei möglicherweise noch an Gewicht zunehmen. Je nach Sportart und Trainingsbelastung, die gering oder intensiv, kurz oder lang sein kann, umfasst der Energiebedarf verschiedener Athleten eine Spannweite von 2000-8000 kcal pro Tag.

Dabei ist es entscheidend, dass die Energiezufuhr ausreichend und angepasst ist. Eine überhöhte Energieaufnahme kann zu einer Zunahme an Körperfett führen, ist andererseits aber auch notwendig zum Aufbau von Muskelmasse. Nicht selten machen junge Sportler die Erfahrung, dass sie zwar intensiv trainieren, aber weder Muskelmasse zulegen noch Trainingseffekte erzielen. Eine Ursache dafür kann die unzureichende Energiezufuhr sein $[3,4]$.

Im Gegensatz dazu stehen Sportler, die teilweise chronisch zu wenig Energie aufnehmen, um ein niedriges Körpergewicht zu halten. Bei diesen Sportlern kann die Energieverfügbarkeit (EV) erniedrigt ein. Die EV errechnet sich aus der Differenz zwischen Energiezufuhr und Trainingsenergieumsatz und sollte nicht geringer als $30 \mathrm{kcal}$ pro kg fettfreie Masse sein, um Veränderungen im Hormonhaushalt wie z. B. reduzierte Insulin-, IGF- und Sexualhormonspiegel sowie erhöhte Cortisolspiegel und damit mögli- 
che negative Konsequenzen für Leistungsfähigkeit und Gesundheit zu vermeiden $[3,5]$. Beispiele für Sportarten, bei denen Athleten ein erhöhtes Risiko für eine geringe EV haben, sind Hochsprung, Skispringen oder Gewichtsklassensportler [5, 6].

\section{Wie viel Protein brauchen Sportler?}

$\nabla$

Neben einer bedarfsgerechten Energiezufuhr ist es wichtig, die Energie sinnvoll auf die Energieträger Kohlenhydrate, Fette und Protein zu verteilen. Da Proteine für das Muskelwachstum erforderlich sind, wird oft postuliert, dass Sportler mehr davon brauchen. Für Freizeitsportler trifft das nicht zu, für sie gelten die Empfehlungen der DGE mit 0,8g Protein pro kg Körpergewicht. Die tatsächliche Zufuhr liegt den Ergebnissen der Nationalen Verzehrsstudie zufolge sogar bei 1-1,2g Protein pro kg Körpergewicht [7].

Ein $80 \mathrm{~kg}$ schwerer Freizeitsportler hat demnach einen Proteinbedarf von $64 \mathrm{~g}$ pro Tag $(80 \mathrm{~kg} \times 0,8 \mathrm{~g} / \mathrm{kg})$. Diese Menge ist problemlos mit der normalen Ernährung erreichbar. Beispielsweise liefert ein Schnitzel oder Steak von $200 \mathrm{~g}$ bereits $40 \mathrm{~g}$ Protein, ein 500 -g-Becher Magerquark enthält ca. $70 \mathrm{~g}$ Protein. Auch Getreide ist reich an Eiweiß ( $\bullet$ Abb.2).

Für Ausdauer- und Kraftsportler gelten etwas höhere Proteinmengen von täglich 1,2-1,7g pro kg Körpergewicht [8]. Für eine Proteinzufuhr von über $2,5 \mathrm{~g}$ pro $\mathrm{kg}$ Körpergewicht gibt es keine wissenschaftliche Grundlage [9-11]. Derart hohe oder noch höhere Zufuhren werden beispielsweise im Bodybuildingbereich erreicht. Generell gilt: Bei ausreichender Energiezufuhr kann auch der erhöhte Proteinbedarf von Leistungssportlern über die normale Ernährung gedeckt werden. Eine zusätzliche Einnahme von Protein- und Aminosäurepräparaten ist nicht nötig [8].

Dennoch gibt es Risikogruppen, bei denen die Proteinzufuhr kritisch werden kann. Dazu gehören Athleten, die auf ihr Körpergewicht achten müssen und bewusst wenig Energie zuführen oder Sportler mit Lebensmittelunverträglichkeiten, etwa einer Laktoseintoleranz. Im Prinzip können auch sie den Proteinbedarf durch eine geschickte Auswahl von Nahrungsmitteln decken. Alternativ sind Proteinshakes in diesen Fällen praktisch - allerdings nicht aus physiologischen, sondern aus organisatorischen Gründen.

Für Sportler spielt nicht nur die absolute Proteinmenge, sondern auch das richtige Timing eine Rolle. Die wenigen dazu vorliegenden Studien weisen darauf hin, dass 15-25g Protein nach einem Krafttraining optimal sind, um die Muskelproteinsynthese anzuregen $[11,12]$. Höhere Dosierungen von 40 oder gar $60 \mathrm{~g}$ Protein, wie manche Hersteller sie anbieten, haben dagegen keinen zusätzlichen Effekt, sondern gehen als Energiesubstrat in den Stoffwechsel ein.

\section{Kohlenhydrate: Brennstoff für die Muskeln}

Für Sportler ebenfalls wichtig sind Kohlenhydrate. Da sie schnelle Energielieferanten sind, galt bis zu den 1990er-Jahren die pauschale Empfehlung, dass Sportler 55-65\% ihrer täglichen Energie in Form von Kohlenhydraten aufnehmen sollen [13]. Insbesondere seit ca. 10 Jahren differenziert man weitaus stärker nach Trainingsphase und -belastung, nach dem Zeitpunkt vor, während und nach der Belastung, der Menge und Qualität der Kohlenhydrate [14]. Diese Detailfragen sind für Gesundheitsund Breitensportler jedoch nicht relevant. Die empfohlene Koh-

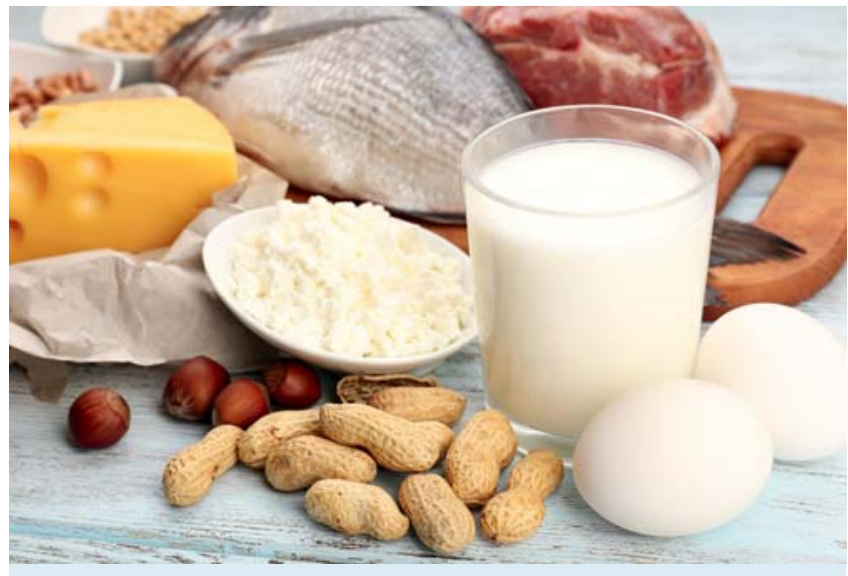

Abb.2 Auch der etwas erhöhte Proteinbedarf von Ausdauer- und Kraftsportlern lässt sich problemlos mit Lebensmitteln decken. Fleisch, Quark, Milch, Eier, Getreide und Nüsse sind gute Eiweißlieferanten. Eiweißshakes oder Aminosäurepräparate sind nur in Ausnahmefällen sinnvoll, etwa bei Sportlern, die ihr Gewicht reduzieren müssen und bewusst wenig Energie zuführen (Quelle: fotolia, @ Africa Studio).

lenhydratzufuhr pro Kilo Körpergewicht steigt dynamisch mit der Dauer der Belastung [14]. Für leichte Trainingseinheiten sind 3-5g Kohlenhydrate pro Kilo Körpergewicht ausreichend; das entspricht in etwa den Empfehlungen der DGE für die Allgemeinbevölkerung. Bei täglich einer Stunde Training erhöht sich der Kohlenhydratbedarf auf 5-7g, bei täglich bis zu 3 Stunden auf 6-10 g, bei extremer Ausdauerbelastung von 4-5 Stunden täglich auf 8-12g Kohlenhydrate pro Kilo Körpergewicht [14]. Das sind sehr große Mengen, die weit entfernt sind von der Ernährung der Allgemeinbevölkerung.

Fazit: Die Empfehlungen für die Kohlenhydratzufuhr sollten fein auf die individuellen Trainingsbedürfnisse abgestimmt werden. Das Training bestimmt, wie viele Kohlenhydrate man braucht. Sie liefern die notwendige Energie, um bei höheren Intensitäten möglichst lange trainieren zu können. Werden Kohlenhydrate dagegen nicht durch Training verbraucht, werden sie in Fett umgewandelt.

Wie wichtig eine ausreichende Versorgung mit Kohlenhydraten für Athleten ist, zeigt eine randomisierte Cross-over-Studie, bei der 7 trainierte Läufer zweimal 11 Tage ein ehrgeiziges Trainingsdesign durchlaufen haben - einmal mit 8,4g, einmal mit 5,4g Kohlenhydraten pro kg Körpergewicht [15]. Dabei wurde die Laufgeschwindigkeit in Abhängigkeit von der Kohlenhydratzufuhr gemessen. In der Phase mit der hohen Kohlenhydratzufuhr konnten die Läufer ihre Laufgeschwindigkeit in etwa halten, während die Geschwindigkeit bei niedriger Kohlenhydratzufuhr abfiel. Die Autoren kommen zu dem Schluss, dass eine höhere Kohlenhydratzufuhr zu einer besseren Leistungsfähigkeit im Training führen kann. Wenn es also im Training und Wettkampf um eine maximale Leistung geht, ist eine optimale Versorgung mit dem Energiesubstrat Kohlenhydrate von besonderer Bedeutung.

Wo genau die optimale Kohlenhydratzufuhr in einer bestimmten Trainingsphase liegt, lässt sich manchmal nur abschätzen. Sie ist aber abhängig vom Gesamtenergieumsatz und sollte in Anlehnung an die Trainingsbelastung auf die individuellen Bedürfnisse abgestimmt werden. Wie stark die Trainingsbelastung und damit die Kohlenhydratzufuhr variieren kann, zeigt das Beispiel der Sportart Leichtathletik: Während eine Marathonläuferin (50 kg) in einer intensiven Phase 400-500g Kohlenhydrate pro Tag be- 


\begin{tabular}{l|l}
\hline $\begin{array}{l}\text { Sportlerin }(50 \mathrm{~kg}) \text { in einer } \\
\text { intensiven Trainingsphase mit } \\
\text { hoher Trainingsbelastung } \\
\text { (z. B. Marathon) }\end{array}$ & $\begin{array}{l}\text { Sportlerin }(60 \mathrm{~kg}) \text { an einem Tag } \\
\text { mit geringer Trainingsbelastung } \\
\text { (z. B. Hochsprung) }\end{array}$ \\
\hline $8 \mathrm{~g} / \mathrm{kg} \mathrm{KG} \times 50 \mathrm{~kg}=400 \mathrm{~g}$ & $3 \mathrm{~g} / \mathrm{kg} \mathrm{KG} \times 60 \mathrm{~kg}=180 \mathrm{~g}$ \\
\hline $10 \mathrm{~g} / \mathrm{kg} \mathrm{KG} \times 50 \mathrm{~kg}=500 \mathrm{~g}$ & $5 \mathrm{~g} / \mathrm{kg} \mathrm{KG} \times 60 \mathrm{~kg}=300 \mathrm{~g}$ \\
\hline $\begin{array}{l}\text { Die Kohlenhydratzufuhr sollte } \\
\text { in etwa } 400 \text { bis } 500 \mathrm{~g} \text { pro Tag } \\
\text { betragen }\end{array}$ & $\begin{array}{l}\text { Die Kohlenhydratzufuhr sollte } \\
\text { in etwa } 180 \text { bis } 300 \mathrm{~g} \text { pro Tag } \\
\text { betragen }\end{array}$ \\
\hline
\end{tabular}

Abb.3 Die Kohlenhydratzufuhr im Hochleistungssport ist keine fixe Größe, sondern abhängig von der Trainingsbelastung.

nötigt, kommt eine Hochspringerin $(60 \mathrm{~kg})$ an einem Tag mit geringer Trainingsbelastung mit 180-300g Kohlenhydraten aus (๑ Abb.3).

Nicht nur die Muskeln nutzen Kohlenhydrate als Substrat, sondern auch das Gehirn. Möglicherweise fällt der Sport leichter, wenn entsprechend kleine Mengen Kohlenhydrate während des Trainings nachgefüllt werden. Insofern könnte dies für den Freizeitsportler sinnvoll sein, denn wer Spaß am Sport hat, wird ihn auch regelmäßiger machen.

\section{Kohlenhydratnachschub: Riegel, Gele, Sportgetränke \\ $\nabla$}

Kleinere Mengen an Kohlenhydraten während des Trainings können die kognitive und körperliche Leistungsfähigkeit verbessern [14]. Bei kurzen Belastungen von bis zu 45 min ist dies noch nicht nötig. Wer 45-75 min trainiert und an sein Leistungslimit gehen möchte, sollte 30-60g Kohlenhydrate aufnehmen, je nach individuellen Vorlieben kann dies mit einem Riegel, einem Sportgetränk oder einem Gel erfolgen. Diese Empfehlungen können aber nur Richtwerte sein. Letztlich müssen Athleten selbst herausfinden, was am besten für sie passt $[14,16]$.

Extremausdauersportler brauchen zum Teil große Mengen (ca. $90 \mathrm{~g}$ pro Stunde) an Kohlenhydraten während der Belastung. Am besten verträglich erscheint eine Mischung von Glukose und Fruktose. Beide Zucker werden im Darm mithilfe unterschiedlicher Carrier (Glukose SGLT1, Fruktose GLUT5) resorbiert. Flutet ausschließlich Glukose im Darm an, ist der SGLT1-Transporter bei Mengen über $60 \mathrm{~g}$ pro Stunde irgendwann gesättigt, die Glukose häuft sich an und kann zu Magen-Darm-Problemen führen. Studien haben gezeigt, dass ein Kohlenhydratmix aus zwei Teilen Glukose und einem Teil Fruktose die Carrier besser auslastet, dabei die Kohlenhydratverfügbarkeit verbessert und die MagenDarm-Verträglichkeit gesteigert ist $[14,16]$.

\section{Low Carb: Kein Konzept für Leistungssportler}

\section{$\nabla$}

Low-Carb-Diäten mit geringer Kohlenhydratzufuhr sind im Fitnessbereich relativ weit verbreitet, aber im Leistungssport in den meisten Fällen kontraproduktiv, da sie Verschlechterungen in den Trainingsleistungen, eine reduzierte Trainingsanpassung und erhöhte Infektanfälligkeit mit sich bringen können. Andererseits kann ein sog. Low-Glykogen-Training in ausgewählten Trainingsphasen zu verbesserten Anpassungsprozessen führen und die Leistungsfähigkeit erhöhen. Erste Untersuchungen zu den Anpassungseffekten bei einem Training mit entleerten Glykogenspeichern wurden 2005 durchgeführt [17]. Eine schwedische
Arbeitsgruppe ließ Personen über 10 Wochen das jeweils linke und rechte Bein zu verschiedenen Zeitpunkten trainieren. Zunächst wurden beide Beine durch einen Beinstrecker belastet. Ein Bein machte den restlichen Tag Pause und wurde erst am nächsten Tag wieder belastet. Das andere Bein wurde nach 2 Stunden mit nahezu entleerten Gkykogenspeichern (keine Nahrungsaufnahme in den 2 Stunden) nochmals belastet und machte dafür am nächsten Tag Pause. Die Belastung erfolgte also einmal mit vollen, einmal mit leeren Glykogenspeichern. Trotz gewisser limitierender Aussagekraft der Studie lässt sich allgemein festhalten, dass das Bein, welches jede zweite Trainingseinheit mit entleerten Glykogenspeichern trainiert hat, im abschließenden Test leistungsfähiger war. Dabei ist zu beachten, dass die Ernährung der Personen mit einem Anteil von 70\% generell aber sehr kohlenhydratreich war.

Darin besteht der grundsätzliche Unterschied zu Low-Carb-Diäten. „Train Low“ wurde zum Schlagwort, die praktische Anwendung aber ist problematisch, wenn es mit Low Carb gleichgesetzt wird. Eine geringe Verfügbarkeit von Kohlenhydraten kann zu einer reduzierten Belastbarkeit im Training und geringeren Trainingseffekten führen. Zum anderen kommt es zu einer vermehrten Glukoneogenese. Das heißt, der Organismus bildet aus Aminosäuren vermehrt Glukose. Damit steigt unter Umständen das Risiko für Infekte und einem Verlust an Muskelmasse. Diese Risiken verschärfen sich zusätzlich bei „Training-Zero“, also chronisch niedriger Zufuhr von Kohlenhydraten [18].

Training in einem glykogenverarmten Zustand kann zu verbesserten Anpassungsprozessen führen und die Leistungsfähigkeit verbessern. Ob die Durchführung eines solchen „trainlow“-Konzepts auch bei gut trainierten Athleten die Leistungsfähigkeit verbessert, ist unklar [14].

Weitere wichtige Themenfelder in der Ernährung des Sportlers wie Flüssigkeitshaushalt, Mikronährstoffe und Nahrungsergänzungsmittel werden an anderer Stelle in diesem Supplement besprochen.

Die Ernährung hat Einfluss auf die körperliche und mentale Leistungsfähigkeit und ist für Leistungssportler eine wichtige trainingsbegleitende Maßnahme. Es handelt sich dabei jedoch nicht um eine Ernährungsform, die für alle Sportler in jeder Situation gültig ist. Die Frage nach der optimalen Ernährung benötigt eine Auseinandersetzung mit der Sportart, der aktuellen Trainingsphase und den individuellen Bedürfnissen und Zielen der Athleten. Die Umsetzung benötigt Zeit, die Eigenmotivation der Sportler und des Fachpersonals, das sich intensiv mit den Ernährungsbedürfnissen von Sportlern auseinandersetzt.

\section{Interessenkonflikt}

$\nabla$

Der Autor hat keinen Interessenkonflikt.

\section{Literatur}

1 Hollmann W, Strüder HK. Sportmedizin: Grundlagen für körperliche Aktivität, Training und Präventivmedizin. 5. Auflage. Stuttgart: Schattauer; 2009

2 IOC consensus statement on sports nutrition 2010. Journal of Sports Sciences 2011; 29 (Suppl. 01): S3-S4

3 Loucks AB, Kiens B, Wright HH. Energy availability in athletes. Journal of Sports Sciences 2011; 29 (Suppl. 01): S7-S15

4 Desbrow B, McCormack J, Burke LM et al. Sports Dietitians Australia position statement: sports nutrition for the adolescent athlete. Int J Sport Nutr Exerc Metab 2014; 24: 570-584

5 Loucks $A B$. Energy balance and body composition in sports and exercise. Journal of Sports Sciences 2004; 22: 1-14 
6 Braun H. Besonderheiten der Ernährung in Sportarten mit Gewichtsklassen. Aktuel Ernahrungsmed 2010; 35: 178-182

7 Max Rubner Institut. Nationale Verzehrsstudie II. Karlsruhe: 2008: https://www.mri.bund.de/fileadmin/Institute/EV/NVSI_Abschlussbe richt_Teil_2.pdf (Zugriff 1.11.2015)

8 Rodriguez NR, Di Marco NM, Langley S. American College of Sports Medicine position stand. Nutrition and athletic performance. Med Sci Sports Exerc 2009; 41: 709-731

9 Phillips SM. A Brief Review of Higher Dietary Protein Diets in Weight Loss: A Focus on Athletes. Sports Med 2014; 44 (Suppl. 02): S149S153

10 Deutsche Gesellschaft für Ernährung. Referenzwerte für die Nährstoffzufuhr. 1. Auflage. Frankfurt am Main: Umschau/Braus; 2000

11 Phillips SM, Van Loon LJC. Dietary protein for athletes: From requirements to optimum adaptation. Journal of Sports Sciences 2011; 29 (Suppl. 01): S29-S38

12 Moore DR, Robinson MJ, Fry JL et al. Ingested proteindose response of muscle and albumin protein synthesis after resistance exercise in young men. Am J Clin Nutr 2009; 89: 161 -168
13 Position of the American Dietetic Association and the Canadian Dietetic Association: nutrition for physical fitness and athletic performance for adults. J Am Diet Assoc 1993; 93: 691 - 696 Review. Erratum in: J Am Diet Assoc 1993; 93: 863

14 Burke LM, Hawley JA, Wong SHS et al. Carbohydrates for training and competition. Journal of Sports Sciences 2011; 29 (Suppl. 01): S17-S27

15 Achten J, Halson SL, Moseley L et al. Higher dietary carbohydrate content during intensified running training results in better maintenance of performance and mood state. J Appl Physiol 2004; 96: 1331-1340

16 Jeukendrup AE. Nutrition for endurance sports: Marathon, triathlon, and road cycling. Journal of Sports Sciences 2011; 29: S91 -S99

17 Hansen AK, Fischer CP, Plomgaard $P$ et al. Skeletal muscle adaptation: training twice every second day vs. training once daily. J Appl Physiol 2005; 98: 93-99

18 Bartlett JD, Hawley JA, Morton JP. Carbohydrate availability and exercise training adaptation: Too much of a good thing? European Journal of Sport Science 2015; 15: 3-12 